\title{
Transatlantica
}

Revue d'études américaines. American Studies Journal

2 | 2014

Aesthetics of Theory in the Modern Era and Beyond / Photographie documentaire

\section{Lewis Hine et le National Child Labor Committee: vérité documentaire et rhétorique visuelle et textuelle}

\section{Anne Lesme}

\section{(Q) OpenEdition}

Journals

Édition électronique

URL : https://journals.openedition.org/transatlantica/7185

DOI : 10.4000/transatlantica.7185

ISSN : 1765-2766

Éditeur

Association française d'Etudes Américaines (AFEA)

Référence électronique

Anne Lesme, « Lewis Hine et le National Child Labor Committee: vérité documentaire et rhétorique visuelle et textuelle», Transatlantica [En ligne], 2 | 2014, mis en ligne le 19 février 2015, consulté le 15 février 2023. URL : http://journals.openedition.org/transatlantica/7185; DOI : https://doi.org/10.4000/ transatlantica.7185

Ce document a été généré automatiquement le 31 janvier 2023.

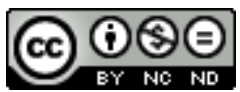

Creative Commons - Attribution - Pas d'Utilisation Commerciale - Pas de Modification 4.0 International - CC BY-NC-ND 4.0

https://creativecommons.org/licenses/by-nc-nd/4.0/ 


\title{
Lewis Hine et le National Child Labor Committee: vérité documentaire et rhétorique visuelle et textuelle
}

\author{
Anne Lesme
}

1 Lorsqu'il photographie les enfants au travail pour le compte du National Child Labour Committee (NCLC) entre 1908 et 1918, Lewis Hine est intimement convaincu que l'aptitude de la photographie à montrer la réalité sert la cause d'une information objective. S'il s'inscrit dans le mouvement progressiste, c'est avec la certitude que la photographie, en apportant la lumière sur une réalité sociale dérangeante - « Let there be light » (Hine, 1909, 357) -, peut faire changer l'ordre des choses et contribuer à engendrer des réformes sociales de grande ampleur en matière de législation du travail, pour des sujets faibles dans le combat social, les enfants. Trois conceptions de la vérité se conjuguent chez Hine qui s'incarnent tout d'abord dans une conviction politique et morale, finalité même de son engagement progressiste; puis dans une considération scientifique et technique sur quoi se fonde confiance totale dans les qualités intrinsèques du médium tout autant que sa méfiance envers l'usage qu'on peut faire de la photographie - «while photographs may not lie, liars may photograph" (Hine, 357). Enfin, la volonté d'une communication optimale le pousse à multiplier les méthodes de diffusion en alliant textes et images pour une efficacité rhétorique toujours plus grande.

2 Or, à cette époque, la croyance en la photographie comme preuve (evidence) s'est affaiblie et sa capacité à représenter fidèlement le réel doit précisément déjà faire ses preuves. D'autre part, lorsque Hine est recruté par le NCLC, on estime à deux millions les enfants de moins de seize ans qui travaillent; le photographe pointe déjà une possible lassitude de la part du public face à des représentations sociales sombres et accusatrices : «Perhaps you are weary of child labor pictures. Well, so are the rest of us, but we propose to make you and the whole country so sick and tired of the whole business that when the time for action comes, child-labor pictures will be records of the past. » (Hine, 357). 
3 Après avoir étudié la manière dont Hine se saisit dans le cadre du Comité (NCLC) d'une référence aux modes de représentation de l'enfance bourgeoise (ne serait-ce que pour s'y opposer), nous verrons quels procédés rhétoriques le photographe va mettre en œuvre, avec le rôle primordial accordé au texte (légendes, rapports...), jusqu'aux affiches et expositions qu'il réalise pour le Comité, ultime étape pour tenter de contrôler au maximum l'interprétation du message, mais aussi une possibilité pour lui de construire la vérité la plus aboutie.

\section{Photographier l'enfant pauvre}

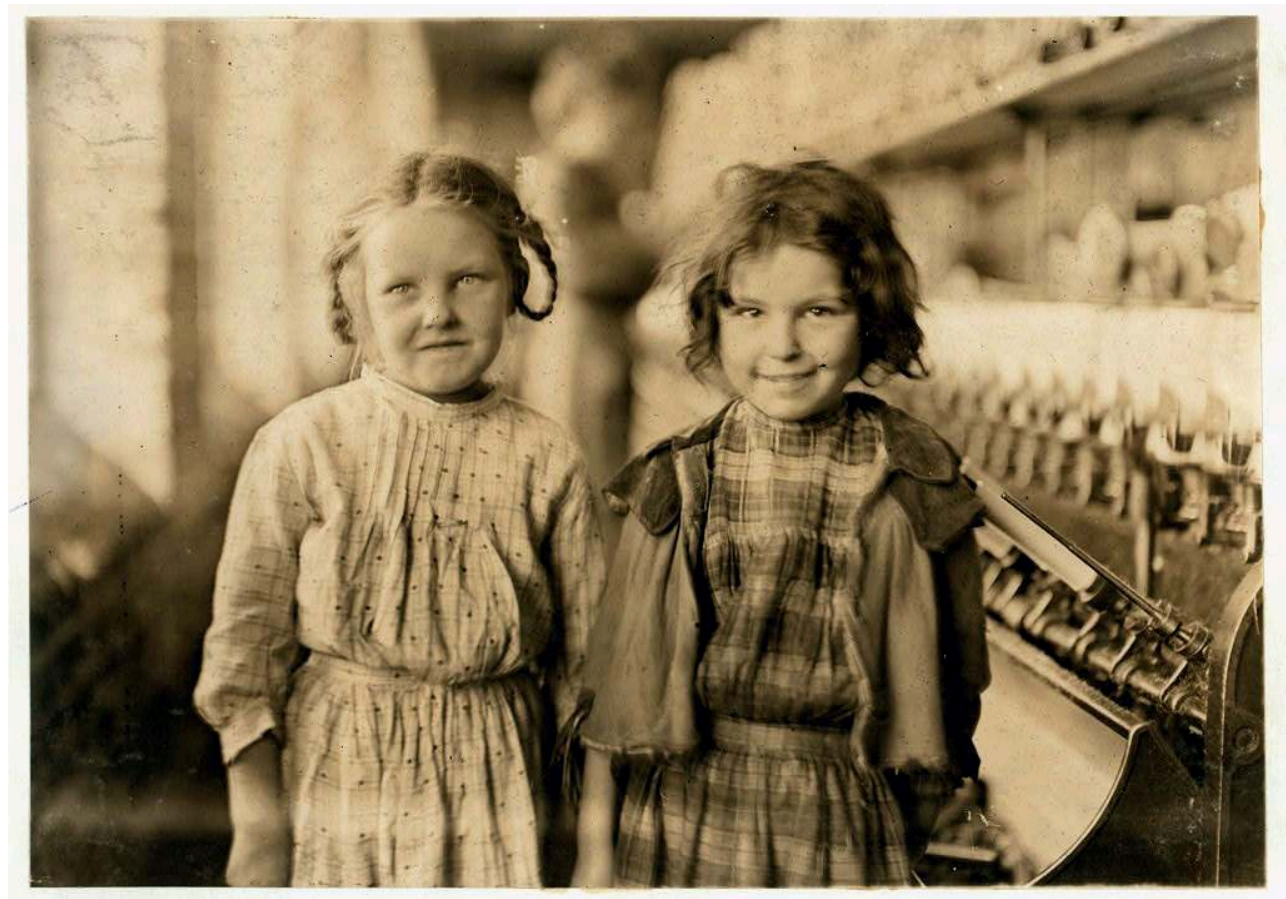

Two of the « helpers » in the Tifton Cotton Mill, Tifton, Ga. They work regularly. Location : Tifton, Georgia. 1909 January. 


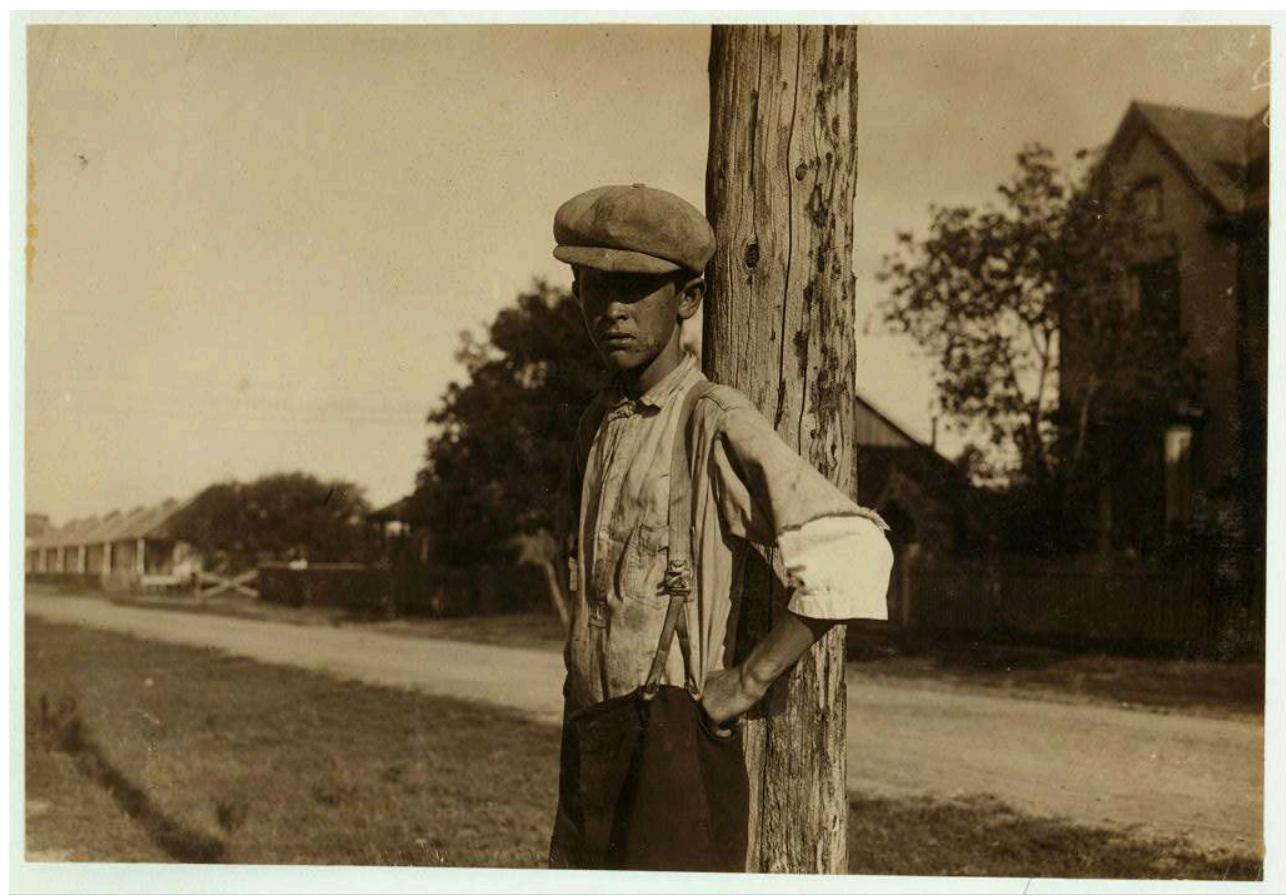

Lewis Hine, John Huggins. Said he is fourteen years old and has been doffing for eight months in the Guadalupe Valley Cotton Mills. 1913 October. ${ }^{1}$

4 Les prises de vue réalisées par Lewis Hine sont le plus souvent frontales, et se font à hauteur d'enfant, en fonction de leur âge, moyen de mettre sur un pied d'égalité le photographe et son sujet dans le cadre d'une photographie à visée mélioriste. Une pratique qui, pour Alan Trachtenberg, permet au photographe d'aller contre la tendance des réformateurs à rabaisser les classes populaires au rang d'objets: « There is then, in Hine's early work, an implicit counterstatement to the Progressive reformist ideology he embraces - a subtle but nonetheless distinct resistance to the tendency of reformers to make objects of their underclass 'cases' « (Trachtenberg, 1989, 206) ${ }^{2}$. A cet égard, Hine se distingue de Riis auquel on a souvent reproché l'approche brutale de la misère et de cette Autre moitié qu'il photographiait ${ }^{3}$. Il convient toutefois de se garder d'une lecture trop univoque du précurseur de la photographie sociale à la fin du XIX à New York, son approche apparaissant en effet le plus souvent bien moins voyeuriste avec les enfants qu'avec les adultes: "These [pictures of children] were the pictures Riis planned from the beginning of his quest, to complete the odyssey from voyeurism to horror to enlightenment, and finally to directed activism » (Hales, 342).

\section{L'enfant pauvre et l'iconographie pictorialiste}

5 Une vérité communément admise dans les milieux aisés, mais aussi très largement dans les classes moyennes à partir du XX $\mathrm{XX}^{\mathrm{e}}$ siècle, est celle des "priceless children » que George Dimock (1993) et Viviana Zelizer (1994) mettent en valeur. L'enfance est reconnue en tant que période de la vie à part entière qu'il convient de protéger. Les photos de Julia Margaret Cameron en Angleterre, tout d'abord, puis d'Alfred Stieglitz, de Clarence White, ou de Gertrude Käsebier, pour ne citer que quelques pictorialistes américains, témoignent de cette imagerie de l'enfance romantique, idéalisée, où spontanéité et individualité semblent souvent s'effacer. Comme le souligne Anne Higonnet, il s'agit d'un enfant « socialement, sexuellement, psychiquement innocent » 
(Higonnet, 1998, 24). L'imagerie, presque irréelle, est vaporeuse et l'angélisme le dispute à l'innocence dans le contexte du pictorialisme. Alors que la photographie devient pour les classes moyennes un mode central de représentation, notamment par l'intermédiaire de l'album de famille, elle remodèle la manière dont les Américains se reconnaissent dans la narration de leur propre vie. La photographie d'enfants oscille entre deux types de vérités, la vérité d'un moment spécifique, et une autre plus large, plus abstraite, telle que celle montrée par Julia Margaret Cameron, qui est moins un portrait que la représentation d'une idée, d'un concept universel. Il en est ainsi des madones à l'enfant de Hine, dont la pause fait explicitement référence à l'histoire de l'art occidental (Beck, 2001, 26), ou bien encore de Happy Days de Gertrude Käsebier (1902), archétype de la représentation photographique pictorialiste, avec son cadrage serré sur l'enfant, la tête des adolescentes coupée, l'exaltation du sentiment de liberté et de l'harmonie avec la nature.

6 Le mouvement de lutte contre le travail des enfants prend le contrepied de cette représentation, tout en utilisant parfois certains de ses codes pour rendre ces photos plus familières, plus touchantes auprès du public, comme Hine l'avait fait pour les immigrants arrivés à Ellis Island, dont il avait cherché à donner une image positive (Sampsell Willmann, 2008).

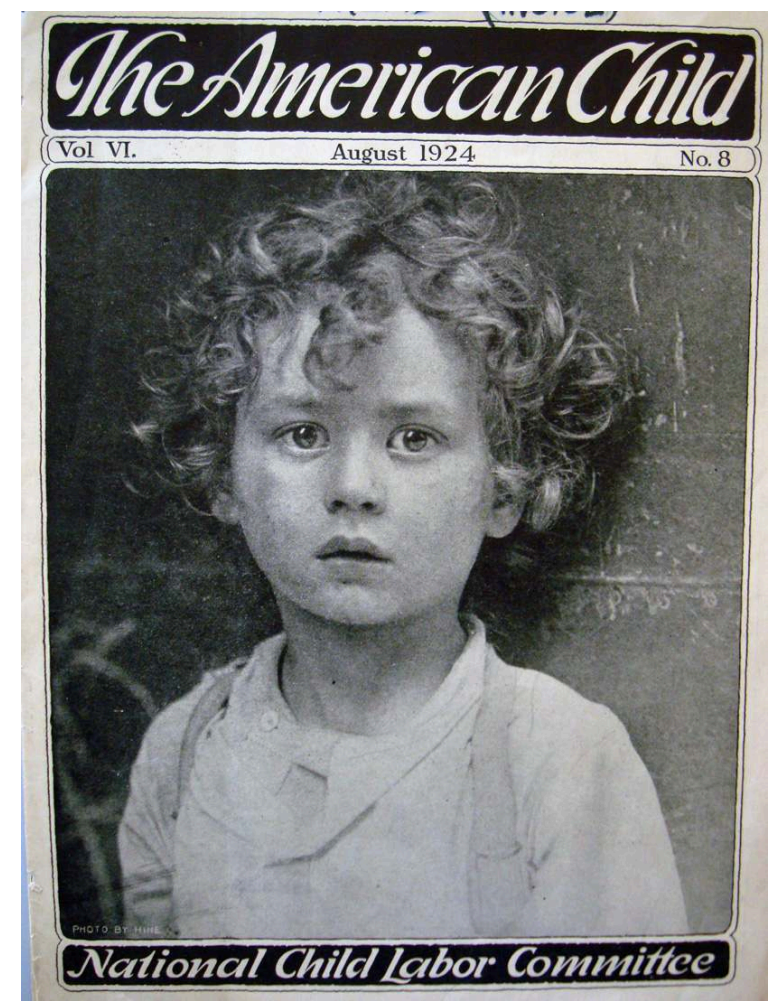

The American Child, April 1924, Nº 8, National Child Labor Committee

7 Comme l'atteste cette photographie publiée dans la revue The American Child en 1924, le mode de représentation de l'enfant pauvre emprunte un certain nombre de ses attributs à l'iconographie pictorialiste qui dépeint le plus souvent des enfants des classes aisées. La tentation est forte en effet de parer l'enfant des rues au visage sali, des atours angéliques du «priceless and Romantic child» (Zelizer, 1994). On pourrait penser que Hine n'a pas été consulté sur ce choix éditorial car il y a une véritable ironie à faire de ce " Gamin de Paris ", photographié en 1918 lors d'un séjour du photographe 
en Europe au service de la Croix Rouge, le symbole de l'enfant américain. Mais si, comme le souligne Alan Trachtenberg, le photographe n'a pas ressenti de contradiction entre l'usage de l'image comme preuve ou comme symbole, alors il n'est pas interdit de supposer que Hine ait avalisé ce choix ou du moins qu'il n'y ait pas été opposé.

D'autres images existent d'enfants américains et non européens, elles ont souvent été utilisées par Hine lui-même, mais l'angélisme - le caractère plus universel - de ce gavroche du vieux continent l'a emporté sur toute autre considération, brouillant les frontières le plus souvent clairement établies entre deux modes de représentation, comme l'explique Sarah Chinn : " The working class child was the polar opposite of the Romantic American child. Whereas the Romantic child was invaluable and outside the mundanities of history, race, gender, and class, the working class embodied the power of those forces. "(37). Car le plus souvent, c'est bien cet enfant aux spécificités détaillées que Hine s'attache à montrer, exposer, décrire, en usant de tous les procédés rhétoriques à sa disposition pour dire le vrai. Il est donc indubitable qu'il existe pour Hine une vérité supérieure à la preuve fournie par la vue photographique.

\section{Absence de contradiction entre l'image-symbole et l'image-preuve}

Il convient tout d'abord de noter que Hine ne répugne aucunement à ce qui apparaîtra comme de coupables manipulations pour les tenants de l'orthodoxie documentaire des années 30, et plus encore pour les adeptes du style documentaire qui faisaient de la neutralité du regard photographique le principe de leur pratique (Lugon, 2002). Le photographe lui-même ne ressent pas de contradiction dans cette manière de faire. "Apparently, Hine felt no discordance between the use of image as evidence and as symbols. But his examples of fashioning a symbolic or connotative discourse for specific images pose the possibility of conflict between, say, the immediate "appeal" of an image and its larger implications » (Trachtenberg, 1989, 207-09). Plusieurs exemples nous sont donnés d'enfants photographiés dont les images sont ensuite utilisées dans un contexte tout autre que celui d'origine. Ainsi parfois le sujet est volontairement grimé et déguisé, comme ce neveu de Hine, George Rich, vêtu de haillons et d'un chapeau de paille alors qu'il appartient à un milieu bourgeois, comme l'a récemment révélé Alison Nordström $(2011,23)$. En janvier 1921, sa photographie est en couverture d'une brochure du NCLC, For Child Labor Day.

10 S'il s'avère nécessaire pour Hine d'asseoir la crédibilité de ses photos, c'est en partie pour répondre aux critiques de ses détracteurs, pas totalement infondées, qui l'accusent de mentir pour parvenir à ses fins. En effet, il convenait pour le photographe de déjouer la vigilance des directeurs d'usines ou des exploitants qui faisaient appel à une importante main-d'œuvre d'enfants. Ainsi, lorsqu'il se faisait passer pour un représentant en machines de filature de coton, l'objectif affiché - représenter l'apologie de l'outil et en montrer sa grandeur en demandant à un enfant de poser dans le cadre photographique, meilleur moyen pour une mise à l'échelle - était à l'inverse de ses intentions réelles.

11 Adepte de la philosophie pragmatique de John Dewey, William James et Charles S. Peirce, Hine s'installe et se revendique comme "photographe social », ainsi qu'il l'écrit sur ses cartes de visite; il est engagé dans une cause qu'il estime juste, puisqu'il s'agit d'informer le grand public, de montrer et de dénoncer le travail des enfants afin de provoquer des changements sociaux. Pour la revue progressiste The Charities and the 
Commons, rebaptisée The Survey en 1908, il est à la fois sociologue et photographe et l'usage qu'il fait d'arguments rhétoriques s'applique aussi bien au langage photographique qu'à l'écriture. Ses notes de terrain sont précises et aussi complètes que possibles : dans un carnet qu'il cache sous son manteau, il consigne le nom, l'âge, la taille, les caractéristiques physiques des enfants, le lieu, les horaires de travail et l'heure, le salaire journalier... Il rappelle la suspicion dont les photographies font l'objet et la nécessité d'avoir un témoin et des documents précis: "My child-labour photographs have already set the authorities to work to see "if such things can be possible". They try to get around them by crying "Fake" but therein lies the value of the data and a witness. My "sociological horizon" broadens hourly ( Gorldberg, 1981, 247-48).

\section{Inventaire des méthodes pour montrer et dire la vérité}

Hine exerce un contrôle considérable sur la présentation et la contextualisation des photos du NCLC. Il s'implique dans la sélection, le découpage, la rédaction des légendes et la distribution des images sur la page pour garantir un effet optimal. Loin d'être simplement descriptives, uniquement linéaires, ses légendes inscrivent les enfants photographiés dans une histoire et dans une série, véritable prélude aux photo essays des années 30. Quant au texte, il s'avère plus que jamais nécessaire pour diminuer la chaîne flottante des signifiés, surtout lorsque les images sont découpées, amputées de leur milieu d'origine et transplantées dans une autre histoire ou bien insérées dans des comparaisons, donc recontextualisées de multiples manières: "Words continued to serve the purpose of anchoring the floating significance of images: Kellogg ${ }^{4}$ and Hine became adept at inventing captions that might undercut the photographic density of the picture and reduce it to a lesson » (Hales, 2005, 431).

Dans l'exigence de vérité du photographe, la dimension pédagogique reste un élément essentiel. D'où les découpages, les nouvelles éditions, retouches ou légendes, au service de l'accroissement du pouvoir didactique des images. Un argument rhétorique retient particulièrement l'attention, celui de la comparaison entre l'enfance au travail et celle de la classe moyenne, établie ici comme norme sociale. Ainsi, dans ce numéro du Child Labor Bulletin, publié en novembre 1912, Hine se livre à deux comparaisons. 


\section{La comparaison}

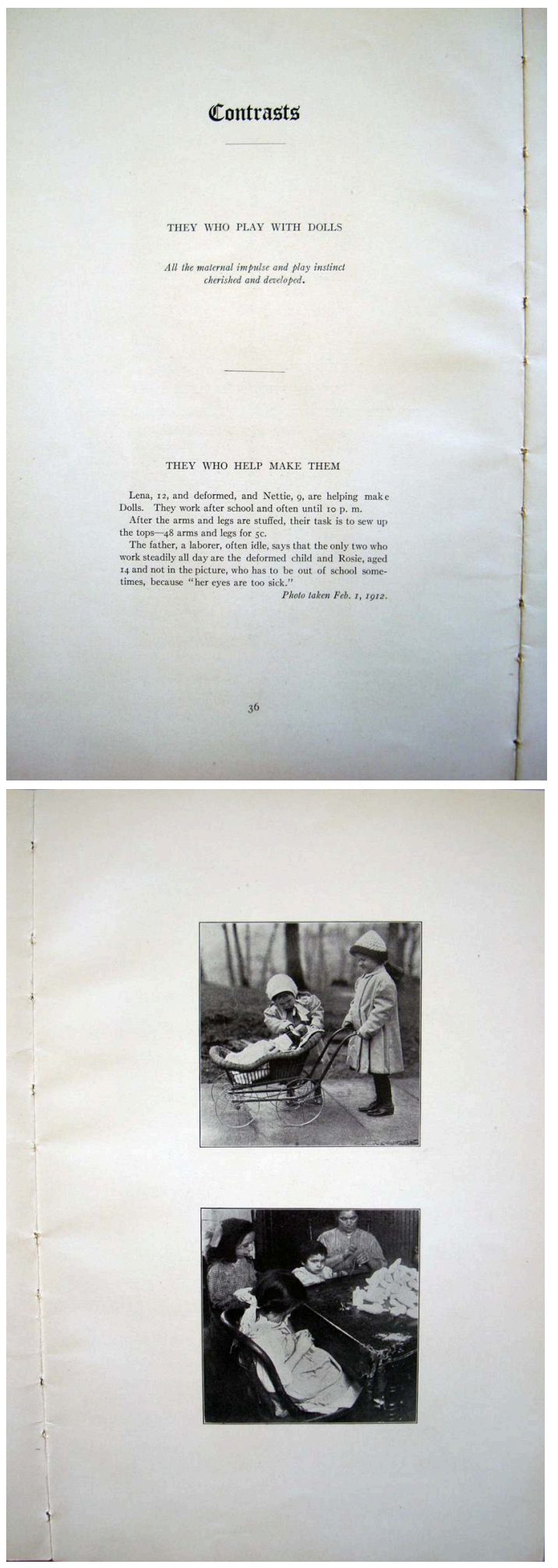

Child Labor Bulletin, Nov 1912

14 La première comparaison a lieu entre les enfants qui jouent à la poupée ("Those Who Play With Dolls») et ceux qui participent à leur fabrication ("Those Who Help Make Them »). Le texte qui fait face à la première photo est laconique mais lourd de sens : 
"All the maternal impulse and play instinct cherished and developed». Alors que la plus grande des petites filles pousse fièrement le landau de la poupée, la plus petite arrange la couverture. La diagonale qui va de la poupée à l'aînée des enfants semble suggérer l'évolution naturelle de la petite fille à la mère attentionnée, le tout dans un paysage de nature, même s'il s'agit d'un parc citadin. Le texte qui accompagne la deuxième image est beaucoup plus long: «Lena, 12, and deformed, and Nettie, 9, are helping make Dolls. They work after school and often until 10 p.m. After the arms and legs are stuffed, their task is to sew up the tops -48 arms and legs for $5 \mathrm{c}$. The father, a laborer, often idle, says that the only two who work steadily all day are the deformed child and Rosie, aged 14 and not in the picture, who had to be out of school sometimes, because 'her eyes are too sick' «. En réduisant la confection des poupées à l'empaillage de quarante-huit bras et jambes et aux coutures à faire, il dit la réduction de l'enfance à l'état d'automates condamnés à des tâches répétitives, fastidieuses - et dangereuses. Alors que la première photo laisse voir une scène en extérieur dans un cadrage aéré, la seconde présente un cadrage serré qui procure un sentiment d'enfermement, voire d'étouffement et laisse deviner la petitesse de l'espace au point que la tête de la mère est coupée. Tous sont au travail, sans exception. 


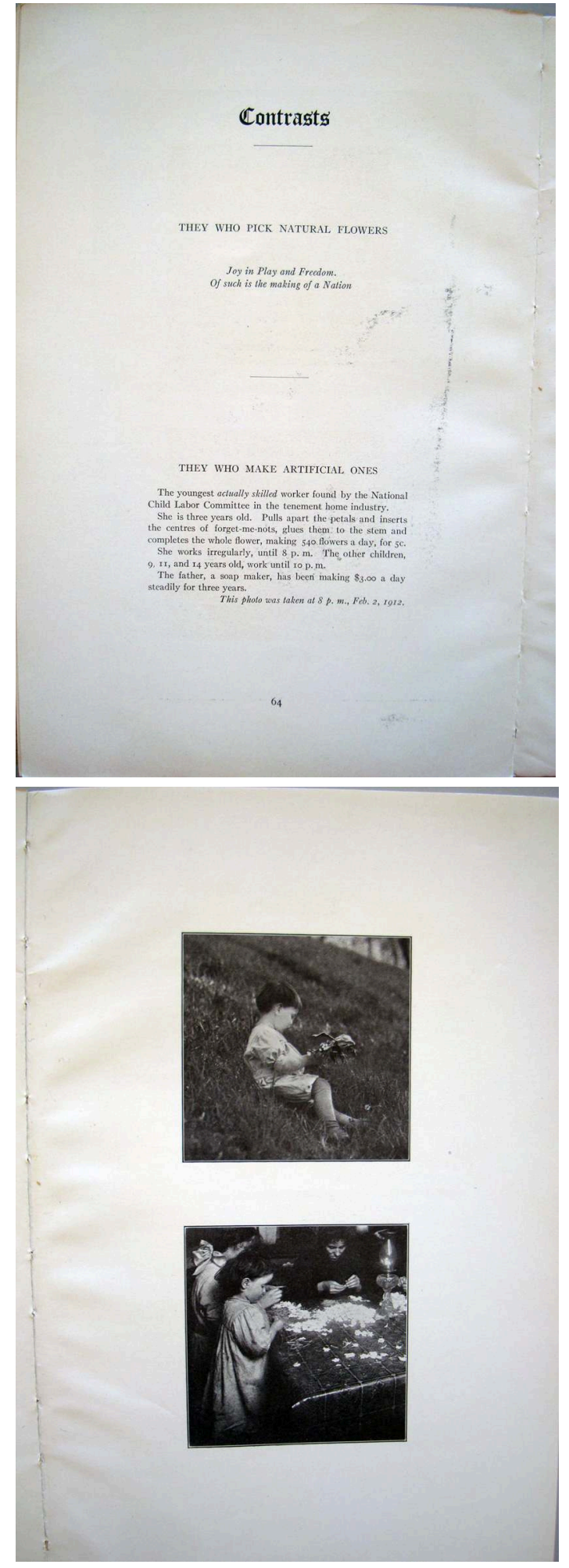

Child Labor Bulletin, Nov 1912

15 La deuxième comparaison s'attache à montrer ceux qui ramassent des fleurs ("They Who Pick Natural Flowers") et ceux qui fabriquent des fleurs artificielles ("They Who Make Artificial Flowers"). A bien des égards, la photographie du petit garçon qui ramasse des fleurs apparaît comme le stéréotype de l'image pictorialiste de l'enfant romantique. Assis dans un cadre champêtre, il est pris de profil, ne regarde pas 
l'appareil et semble insouciant. La légende montre une analogie entre sa santé, son bien-être et celui de la nation tout entière, selon un procédé rhétorique fréquent : « Joy in Play and Freedom. Of such is the making of a Nation ». Son nom ne figure pas, pas plus que la date; tout comme pour les petites filles au landau, ces enfants n'ont pas vocation à avoir une identité, ils sont intemporels, incarnant à eux seuls l'idée que l'on se fait de l'enfance.

En contrepoint, une petite fille du même âge penche la tête dans un univers clos et exigu. Elle ne contemple pas un objet de plaisir mais les pétales de fleurs artificielles qu'elle s'applique à assembler avec sa mère. Elle n'est pas assise mais debout. La légende nous dit «She is three years old ». D'autres frères et sœurs sont présents mais il n'y est pas fait référence explicitement : « The youngest actually skilled worker found by the National Child Labour Committee in the tenement home industry. She is three years old. Pulls apart the petals and inserts the centres of forget-me-nots, glues them to the stem and completes the whole flower, making 540 flowers a day, for $5 \mathrm{c}$. She works irregularly, until 8 p.m. The father, a soap maker, has been making $\$ 3.00$ a day steadily for three years. This photo was taken at 8 p.m., Feb. 2, 1912 ». Le contraste qui nous intéresse ici est aussi celui des fleurs naturelles, authentiques, faisant de la nature le lieu de prédilection de la classe moyenne qui, en revanche, laisse aux enfants des classes populaires qui travaillent le soin d'arranger pour eux sous une lumière au kérosène des fleurs artificielles, fausses, qui alimentent une société avide d'artifice. Ici figurent la date, mais aussi l'heure de la photographie, huit heures du soir; heure à laquelle la petite fille devrait être au lit. L'argument utilisé est ici que le travail des enfants déroge à l'ordre naturel des choses. D'un côté donc, toute l'emphase pictorialiste vise à planter le décor d'une enfance idéale, de l'autre, une vue centrée sur la misère se présente comme la preuve du contraire.

\section{La force rhétorique du texte}

17 La force rhétorique de Hine se situe aussi dans son texte et le caractère parfois très incisif de son style, encore peu étudié. A la manière de Riis, il prend le lecteur ou le spectateur à partie afin de rendre visible ce qui est caché, en instaurant un dialogue avec lui : "Come out with me to one of these canneries at 3 o'clock in the morning" (The Child Labor Bulletin, 2, 1913), écrit-il au sujet du travail des enfants dans les conserveries. La force de l'information factuelle seule ne suffit pas, c'est dans l'acte de communication que se situe l'efficacité de son message social. Tout est fait pour que le public garde les yeux grand ouverts face à cette vérité qui dérange, parce qu'une partie de celle-ci s'affiche au grand jour, comme c'est le cas des petits boulots de rue, en particulier avec les petits vendeurs de journaux, familiers au regard des passants.

Le ton est parfois celui d'une simple narration faite d'euphémismes pour décrire la terrible banalité des accidents du travail qui handicapent les enfants à vie. Souvent, l'ironie cache mal la colère dans une rhétorique puissamment didactique, comme lorsqu'il évoque le monde industriel et la préoccupation du capitalisme pour les coûts : "with its usual unselfish, altruistic spirit». Plus loin il fait référence aux nombreuses tâches qui attendent les enfants: « Did I say tasks?-Not so- they are 'opportunities for the child and the family to enlist in the service of industry and humanity' ». Enfin, il évoque en ces termes les salaires: "Of course they must accept with cheery optimism the steady decline in wages that inevitably follows in the wake of homework. Isn't it 
better anyway for everyone to be working instead of expecting father to do it all? » (The Child Labor Bulletin, May 1914).

19 Cette ironie se retrouve dans divers supports de communication, comme cette photographie de Hine qui sert le propos d'un journaliste raillant la "philanthropie » mise en avant par les patrons d'usine qui emploient des enfants. Elle est en phase avec de nombreux dessins de presse, comme celui de cet enfant à la taille minuscule qui fait face à son juge à la barbe blanche et doit répondre de l'absurdité d'un monde qui défend encore le bien-fondé du travail des enfants au nom de son bien-être: «The CHARGE : Being Born ; the VERDICT : Guilty ; the SENTENCE : Hard Labor for Life ».

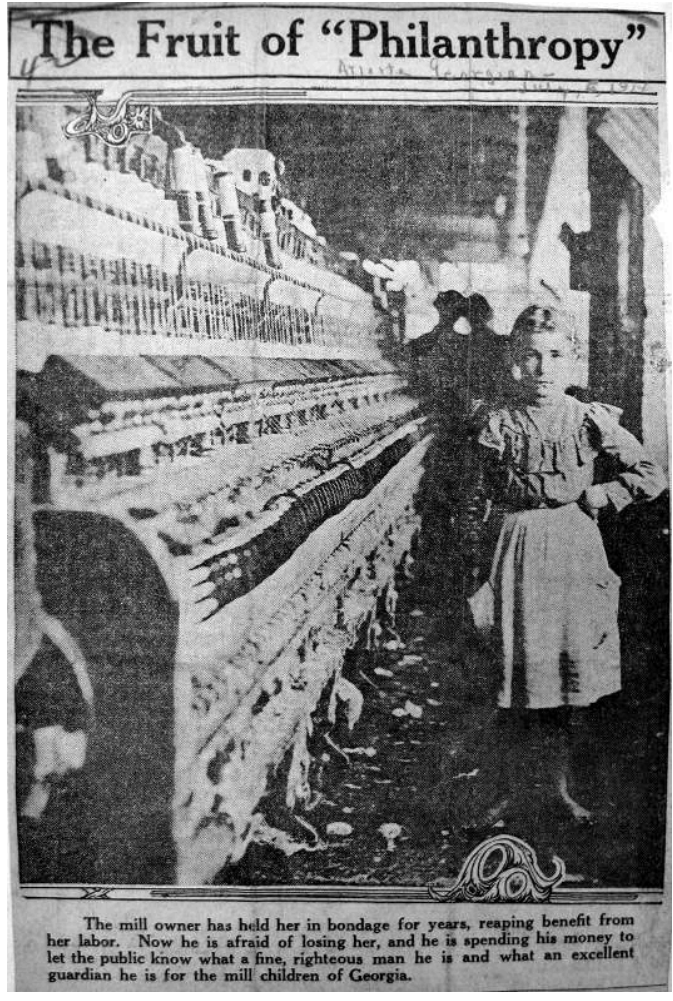

Atlanta Georgia, July 6, 1917 


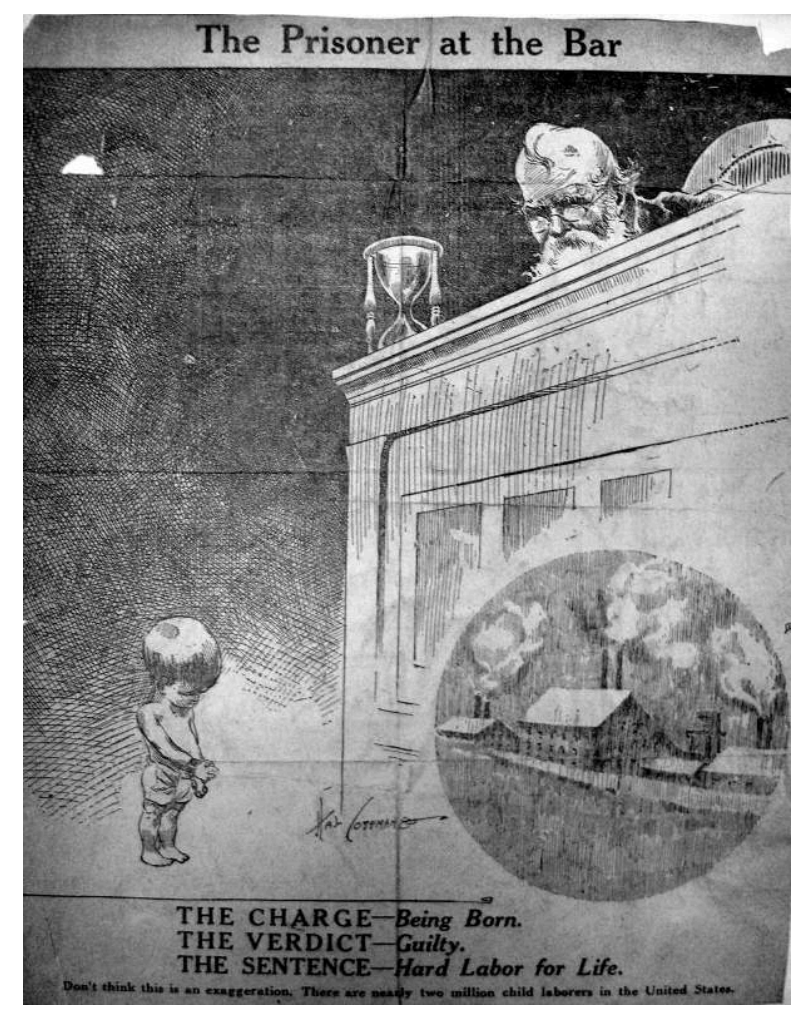

Chicago American, February 10, 1914

20 À mesure que le photographe progresse dans ses investigations et la mise en valeur éditoriale de ses images et des idées défendues par les Progressistes, il développe une communication de plus en plus complète où textes et images se répondent comme en témoigne la série des Time Exposures.

\section{Time Exposures : un message sensible et efficace pour un public ciblé}

21 Ces encarts conçus par Hine ont été publiés dans The Survey en 1914 et 1915 au moment où le magazine expérimentait divers formats de présentation pour mieux communiquer avec son lectorat. Avec ce nouvel agencement des photos, Hine continue à expérimenter de nouveaux modes de communication pour accroître l'efficacité de son message et c'est ainsi qu'il réalise plusieurs photomontages, "a graphic process of juxtaposing several images to achieve new depth of meaning " (Trachtenberg, 1977, 131). Il y ajoute un texte à forte valeur documentaire. Bien plus qu'une légende, les textes de Time Exposure sont un mélange de témoignages, de comparaisons, de contrastes. 


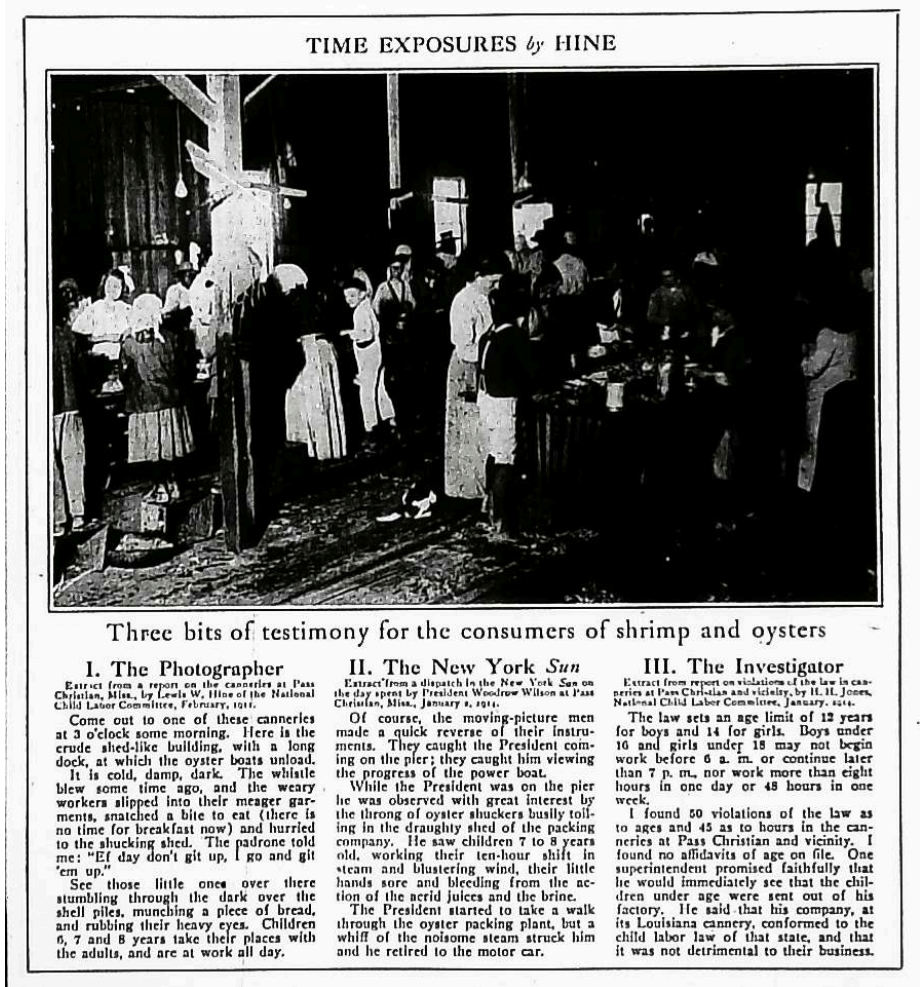

"Three bits of testimony for the consumers of shrimp and oysters », The Survey, Feb. 28, 1914

Le message ci-dessus (The Survey, Feb. 28, 1914, 663) s'adresse textuellement et visuellement aux consommateurs de crevettes et d'huitres. Le plan d'ensemble d'une conserverie montre des travailleurs de tous âges, hommes et femmes, petites filles et petits garçons. De manière singulière, le texte proposé en dessous donne à lire trois extraits qui sont autant de témoignages de sources différentes, et qui s'adressent directement aux consommateurs, c'est à dire au dernier maillon de la chaîne de ce commerce. Loin de se contredire, les récits du photographe, du journaliste du New York Sun et de l'enquêteur du NCLC, conjuguent leurs points de vue pour dénoncer avec la rhétorique qui leur est propre les conditions de travail de cette main d'œuvre parfois âgée de six, sept ou huit ans. Hine s'adresse, comme souvent, directement à son lecteur qu'il semble prendre à partie, et livre le témoignage d'un acteur à qui le 'padrone' va jusqu'à livrer une confidence : "Come out to one of these canneries at 3 o'clock some morning. [...] It is cold, damp, dark. [...] The padrone told me: 'Ef day don't git up, I go and git 'em up' ». Les enquêteurs chiffrent les violations de la loi observées dans cette conserverie: "I found 50 violations of the law as to ages and 45 as to hours in the canneries at Pass Christian and vicinity ». Et le récit le plus poignant, ou révoltant, vient peut-être du journaliste du New York Sun qui, après avoir souligné les dommages corporels causés par les conditions épouvantables dans lesquelles travaillent les enfants, évoque l'inconfort olfactif du Président démocrate Woodrow Wilson qui choisit de se retirer dans sa voiture :

He [President Wilson] saw children 7 to 8 years old, working their ten-hour shift in steam and blustering wind, their little hands sore and bleeding from the action of the acrid juices and the brine. The President started to take a walk through the oyster packing plant, but a whiff of the noisome steam struck him and he retired to the motor car. 


$$
\begin{aligned}
& \text { - photographe, journaliste, enquêteur - au cœur de la campagne des progressistes } \\
& \text { envers l'opinion publique. }
\end{aligned}
$$

\section{Images et poésie}

De manière ponctuelle, il arrive que les photographies de Hine soient accompagnées de poèmes ou de chansons. "Little Brother" (The Survey, vol. 34, May 8,1915, 134) de Florence Ripley Mastin offre un bel exemple de rhétorique poétique, à l'efficacité iconographique et textuelle novatrice.

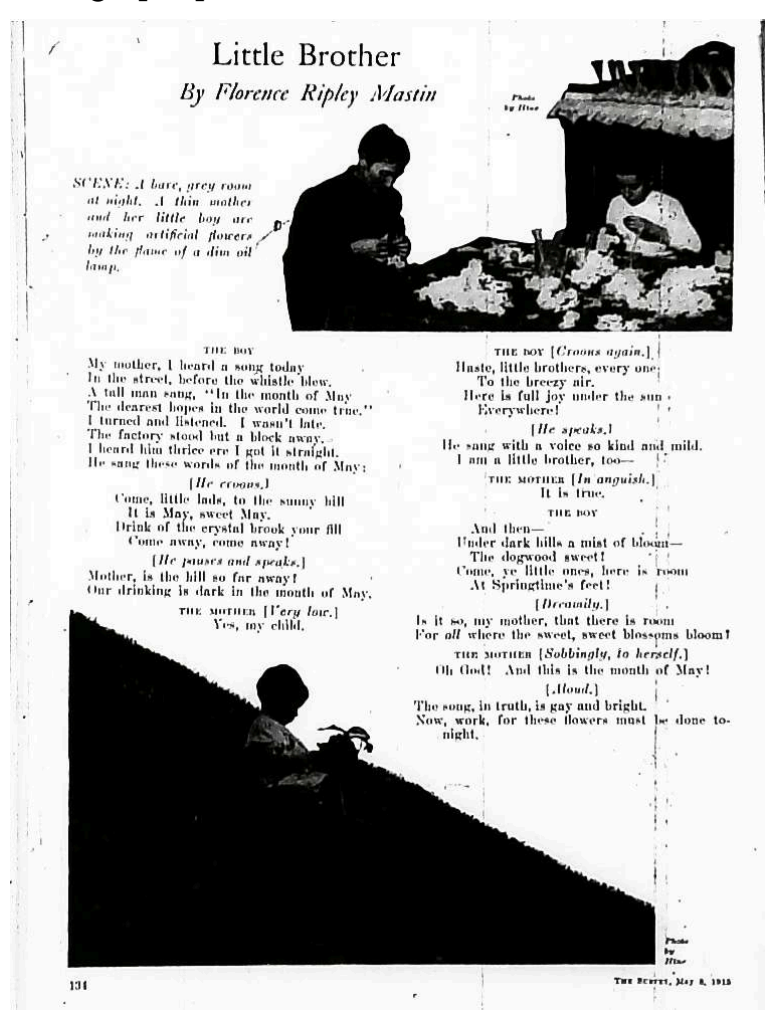

«Little Brother», The Survey, May 8, 1915

Hine réitère un mode de mise en page des photographies sur une pleine page montrant ceux qui fabriquent des fleurs artificielles - une mère et son fils - et celui qui cueille des fleurs fraîches sur la colline par une belle journée de printemps. L'image du petit garçon sur la colline rappelle les clichés pictorialistes des contemporains de Hine (Gertrude Käsebier notamment) ; âgé d'un ou deux ans tout au plus, il se fond dans un paysage graphiquement très ordonné pour encadrer en bas à droite la mise en page du Survey. Vêtu de blanc, tout comme son "Little Brother » occupé dans la diagonale opposée, en haut à droite de la page, à monter les fleurs artificielles à la lumière d'une lampe à huile qui éclaire faiblement, il est, tout comme lui, très concentré. Le texte est une alternance de dialogues entre la mère et le petit garçon qui travaillent et de vers qui se fredonnent, chanson entendue par le petit travailleur dans la rue le jour même. Véritable ode à la nature et au mois de mai, elle rappelle les valeurs de fraternité, quelles que soient son origine et sa condition, un message que l'enfant comprend vite et fait sien. Mais le dialogue rappelle aussi, par la voix de la mère, une réalité bien plus difficile au quotidien : le travail attend et doit être fini dans la nuit. Le mot qui termine 
ces vers («tonight ») renvoie à l'obscurité mainte fois dénoncée par les Progressistes, et s'accorde également avec les deux diagonales qui émergent de la mise en page : la pente descendante de la colline à gauche et la diagonale qui lie les deux « petits frères » dans le sens opposé, ce qui revient métaphoriquement à nier l'enfance.

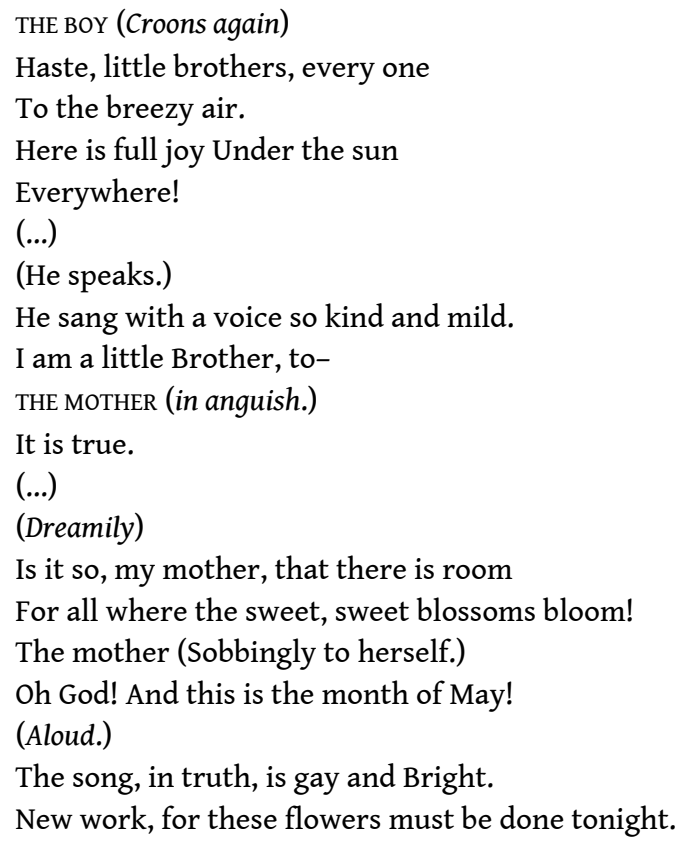

L'expérience du Time Exposures montre une complexification du rapport images/textes avec les ressources nouvelles qui ne font plus seulement des textes une simple légende : les photographies sont parties intégrantes d'un processus descriptif de démonstration. En ce sens, l'ultime étape des moyens rhétoriques utilisés consistera dans la confection des affiches.

\section{Les affiches : document et pratique publicitaire}

Avec la fabrication de ses affiches, Hine pousse à l'extrême la logique et l'analyse qui sous-tendent sa croyance en une efficacité plus grande de la photographie par rapport à la réalité car " les intérêts divergents ont été supprimés » (Hine, 1909, 356) du cadre sélectionné. Il s'agit bien ici de sélection, de découpage du réel au moment du cadrage, logique poussée jusqu'à l'extrême avec la constitution des affiches. Mais Hine va encore plus loin: "The photograph has an added realism of its own; it has an inherent attraction not found in other forms of illustration » $(1909,356)$. Stephen Dane Iseman résume ainsi ce qui différencie l'approche de Hine pour Time Exposures et les affiches qu'il conçoit :

These exhibition panels differed somewhat from his « Time Exposures » in that they were less obtuse in approach than his other text-and-photograph combinations, used fewer words, and used more graphic devices to tie the photographic and textual elements together. The posters and exhibition panels that Hine made typically allowed for less easy alternative readings since the words, though fewer in number, were set in larger and bolder type and made the intention of the presentation more immediately obvious to the viewer. The primary difference in this form may have been that it allowed Hine to reach yet another audience with the National Child Labor Committee's child labor reform messages. (Iseman, 1993, 204-05) 
1914, Hine fonde un département du NCLC spécialisé dans la conception de panneaux d'expositions et l'organisation de leur diffusion. Durant cette seule année, il organisera onze expositions qui tourneront dans cinquante villes (vingt Etats) et dans seize lieux différents dans la seule ville de New York (Goldberg, 1999, 18). Il estime alors qu'il est judicieux et fort adapté de récupérer les méthodes éprouvées de la réclame, utilisées par les milieux économiques qu'il dénonce. Il apparaît également qu'il s'inspire des méthodes des caricatures et dessins qui circulent en grand nombre et dont nous avons vu un exemple (2.2.), l'influence semblant d'ailleurs tout à fait réciproque. L'efficacité de la photographie sociale, telle qu'elle est utilisée en particulier dans les affiches, se joue donc dans l'alliance du document et de la pratique publicitaire (Lugon, 2007, 384). Avec des affiches faites de montages, Hine développe probablement la séquence la plus contrôlée de son travail: "La photographie prend force d'élément discursif au sein d'une chaîne argumentative restreinte » (Lugon, 386). L'usage des mots et de l'image - dont la polysémie est par là même réduite -, s'ajoute à celui de leur mise en scène.

Pour son livret de l'exposition consacrée au travail des enfants paru en juin 1914, Hine réalise une série d'affiches qui lui permet de combiner toutes les recettes qu'il s'est attaché à mettre en œuvre. Il se fait ainsi l'ardent promoteur d'une sur-vérité photographique qui, laissant de moins en moins de place à la chaîne fluctuante des interprétations, lui apparaît irréprochable. Dans « High Cost of Child Labor », tous les arguments des Progressistes mis en avant contre le travail des enfants sont résumés en trois points faciles à retenir et à comprendre: «1. It Costs the Child; 2. It Costs Industry; 3. It Costs Society ». L'argument se termine par la prise à partie du public : «Can we afford child labor?».

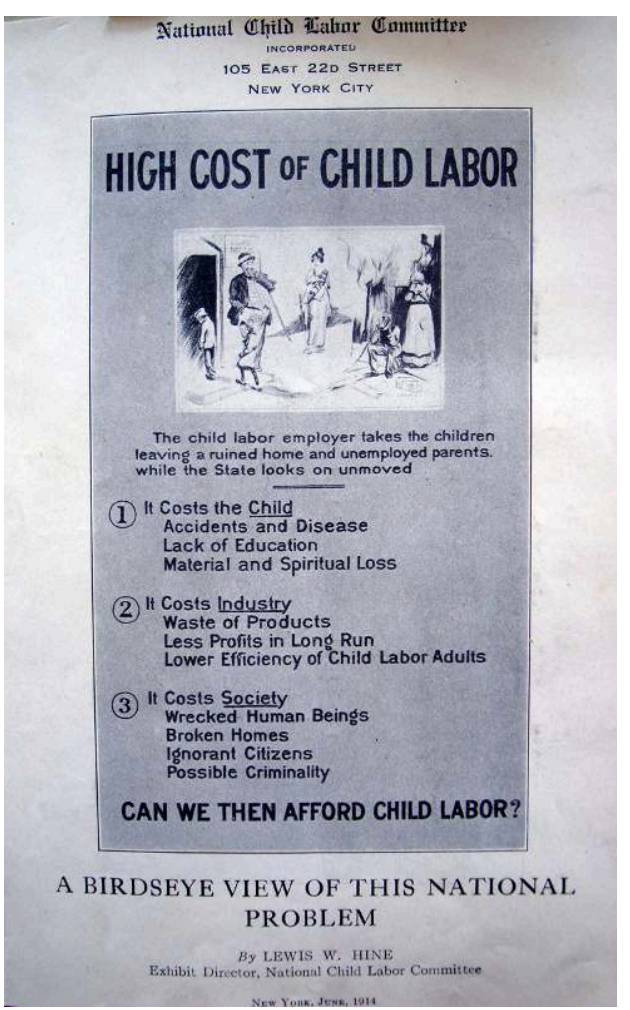

High Cost of Child Labor, «A birdeye view of theis national problem », by Lewis Hine, National Labor Child Committee, 1914

Transatlantica, 2 | 2014 


\section{ACCIDENTS}

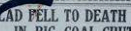

"Children are not

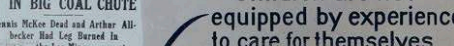

in modern industry"

AND SO THEY PAY

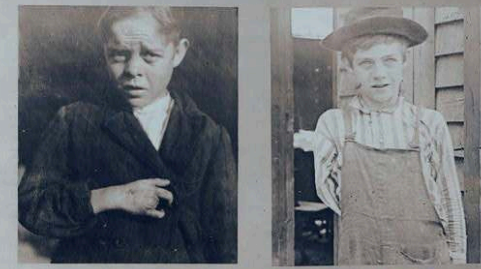

WITH A MAIMED LIFE

Three times as many industrial accidents occur to children as to adults

EMPLOYMENT OF CHILDREN IS DUE TO

IGNORANCE

GREED

NECESSITY

OF INDUSTRY AND PARENTS

ARE ANY OF THESE REASONS WORTH A CHILD'S LIFE?

Exhibit panel, [1913 or 1914?]

L'affiche sur les accidents du travail est remarquable par la nature des photographies utilisées. En effet, jusqu'ici, Hine avait privilégié l'utilisation d'images visant à préserver une image non pathétique de l'enfance. Or, bien des clichés utilisés dans ces affiches font mentir nombre de spécialistes de Hine qui, à l'instar d'Alan Trachtenberg, soulignent la puissance de vie qui se dégage des enfants et qui va à l'encontre du délabrement de leur environnement: « His children have savvy-savoir faire, a wordly air. They have not succumbed. Their spirit is at odds with their surroundings. And this contradiction fills the pictures with an air of tension-not between such abstractions as 'suffering, helpless children' and 'a brutal system' but between living creature and a very particular fate » (Trachenberg, 1977, 131). Il ne s'agit pas ici de remettre en cause cette analyse mais de souligner qu'elle ne correspond pas à ce que ces affiches donnent à voir. D'une part, l'environnement photographique immédiat a été tronqué, ne laissant pas de possibilités de "tension », mais permettant au texte, plus puissant que jamais, de déterminer l'interprétation; d'autre part les visages et les corps choisis servent le propos sans équivoque: enfants mutilés, corps vieillis prématurément, regards hagards, etc. La deuxième image figurant dans l'affiche a certes déjà été déjà utilisée dans le Pittsburgh Survey dès 1909, mais ici la contiguïté avec celle d'un enfant aux doigts accidentés et coupés ajoute à l'effroi ressenti. Une autre de ces affiches a été abondamment commentée, « Making Human Junk » : 


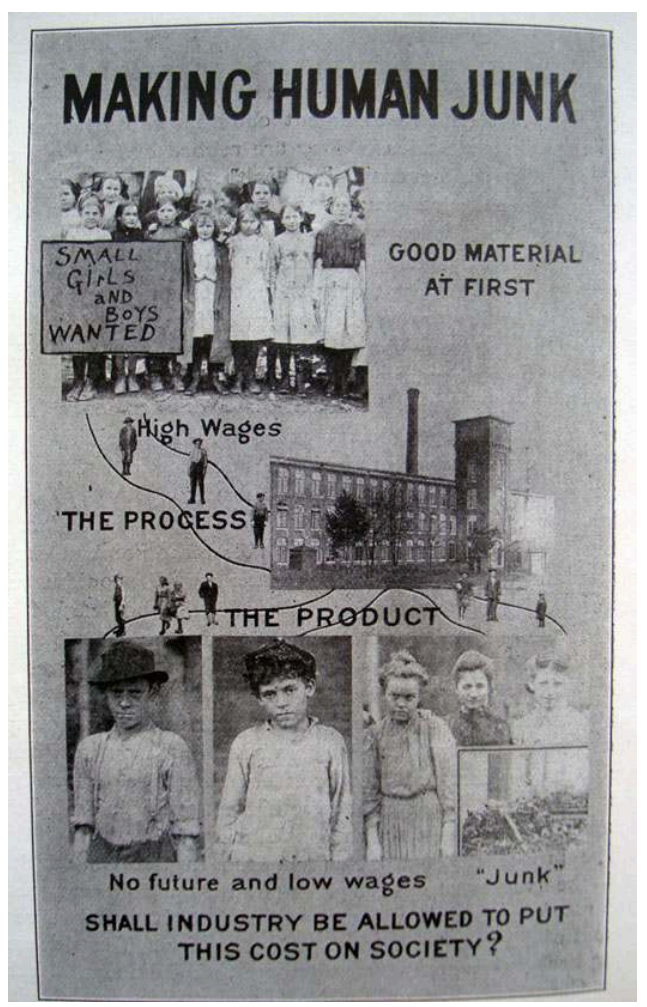

Exhibit panel, [1913 or 1914 ?]

Le montage photographique est plus cruel que chaque photo prise individuellement. La plupart des photos originales ont été coupées. Arrachés à leur environnement photographique d'origine, sans les autres enfants qui les entourent, les visages et les corps de cette affiche font figure de fantômes, effrayants objets de pitié. En observant l'une des photographies utilisées, celle de jeunes ouvrières dans une usine de textile, on peut voir que les deux jeunes filles de gauche, dont l'une esquissait un sourire, ont été supprimées du nouveau cadre. Hine a privilégié le personnage central, symbole d'une croissance à l'usine qui fait ressembler cette très jeune fille à une vieille femme aux épaules voûtées et au visage las. Cependant, la puissance de cette affiche réside davantage dans le dispositif didactique utilisé par Hine, la transformation d'une " matière première » de qualité en un " produit » dénué de valeur, que dans le pouvoir démonstratif des seules images. Ainsi, comme le souligne Didier Aubert : «La "vérité" photographique réside certes dans l'"exactitude" mimétique et l'indéniable fonction probatoire du medium, mais aussi, et surtout, dans ses capacités à intervenir sur les réalités sociales par la saisie et l'évaluation critique. » (Aubert, 2000, 242). 


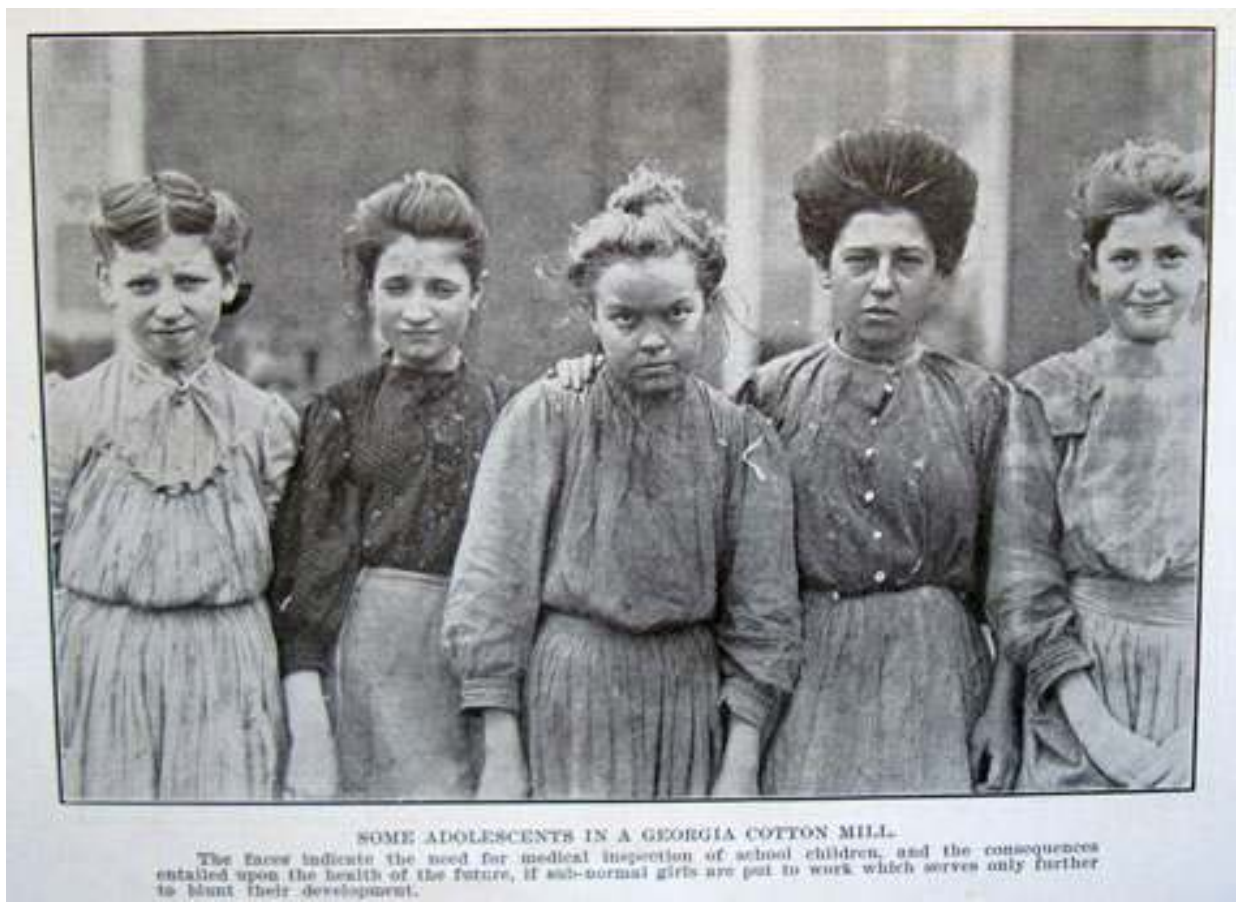

The Survey, «Southerners of Tomorrow », Oct 2, 1909

Les mots et les images se répondent, précurseurs des photo-stories des années 30 (Kaplan, 1989). Pour Miles Orvell: « Hine would compose a sentence which 'reads' both visually and verbally, the words directly supporting the images, creating a kind of primitive picture-story » (Orvell, 2003, 75). La force de persuasion de chaque étape est d'autant plus puissante que ces situations particulières fonctionnent par analogie avec la société. Le développement des enfants avec des activités appropriées (jeu, éducation, protection et affection des parents) est perçu comme crucial pour l'avenir de la nation. Ici, c'est une preuve par l'absurde qui est faite.

Dans l'affiche qui suit, la photographie des jeunes ouvrières est utilisée une fois encore. 


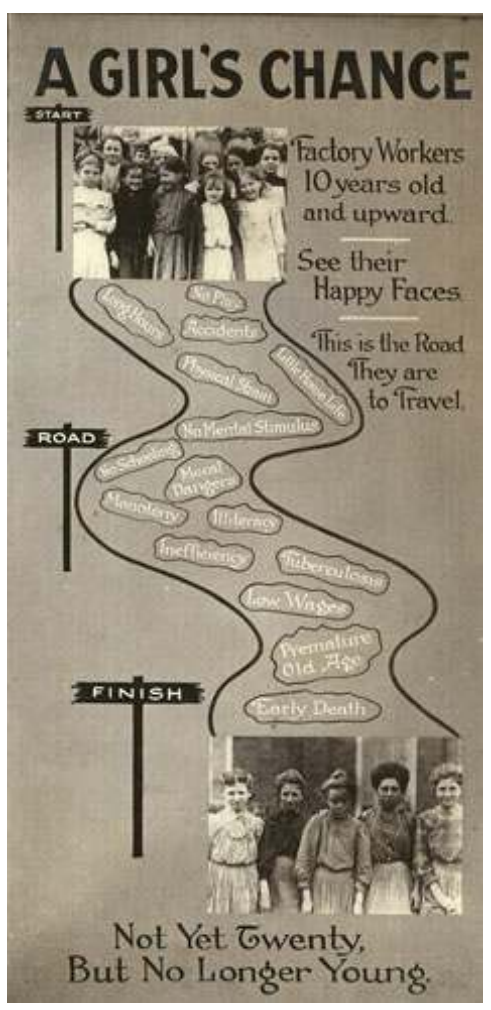

Exhibit panel, [1913 ?]

Intitulée «A girl's chance », cette affiche fait partie d'une exposition pour le NCLC en 1913 et le même argument du vieillissement précoce est utilisé. Cette fois, ce qui est montré dix ans plus tard, car les jeunes filles sont âgées de vingt ans, c'est que désormais, elles «ne sont plus jeunes ». Elles n'ont pas vécu l'âge de l'enfance et à vingt ans, elles paraissent beaucoup plus âgées, ne sourient plus, et si cela arrive à l'une d'elles, elle en est extrêmement gênée. La voie métaphorique que le photographe a tracée ici (du début à la fin) peut être lue comme une synthèse de tous les arguments utilisés par les réformateurs progressistes contre le travail des enfants. Pour le travail en bas âge : «Domicile familial exigu », «Pas de scolarisation », «Pas de jeu », « Pas de stimulation intellectuelle»; "Horaires longs ", "Dangers moraux», "Monotonie », «Illettrisme », «Inefficacité», «Bas salaires»; « Accidents », «Stress », «Tuberculose », "Vieillissement précoce », " Mort prématurée ». La route tortueuse communique clairement une vision de l'enfance comme une période de développement vers l'âge adulte, la maladie et la mort. Le texte et l'image se renforcent ici mutuellement et dirigent, guident et contrôlent plus ou moins l'interprétation du spectateur.

En dirigeant le département des expositions du NCLC, Hine s'engage également à étendre les moyens de communication de l'organisation: tracts, affiches, reportages institutionnels et magazines, brochures, livres. Pour la Panama Exposition 1915, à San Francisco, il prépare vingt-cinq panneaux axés sur deux thèmes principaux : « The High Cost of Child Labor » et "What Are We Going To Do About It?». Selon le Child Labor Bulletin: "The exhibit handbook, amplifying the statements of the panels, which is reprinted in this Bulletin will be for sale " (The Child Labor Bulletin, 7, February 1915). Hine énumère l'ensemble des arguments relatifs aux mouvements de réforme dénonçant le travail des enfants et les condense en des prises de position visuelles et 
textuelles qui sont autant de messages simples et didactiques. L'argumentation de Hine qui accompagne les affiches se termine ainsi :

For the child it is a delusion from every point of view.

For industry it is a fallacy-wasteful, unprofitable, and inefficient.

For society it is a menace-wrecking lives, breaking homes, and ruining citizens.

WE CANNOT AFFORD CHILD LABOR. IT COSTS TOO MUCH. (9)

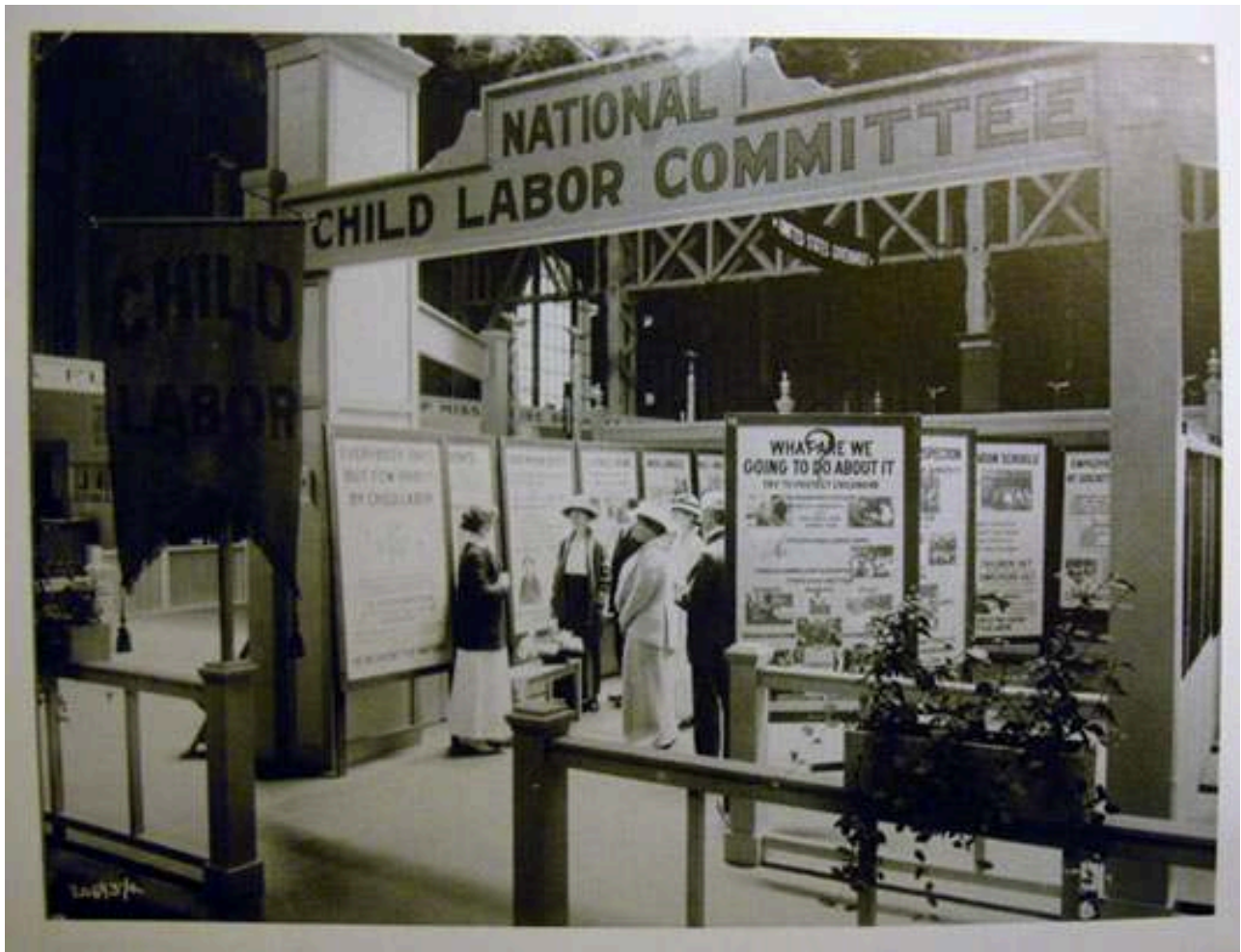

NCLC, The Pan-American Exhibition, San Francisco, 1915

\section{Conclusion}

Pour Hine, la vérité du message, la réalité qu'il s'attache à montrer pour la dénoncer, est une première étape. Son tour de force, en tant que photographe et réformateur social, réside d'une part dans les qualités intrinsèques de ses images d'enfants, d'autre part dans les dispositifs de communication qu'il met en œuvre et qui questionnent sa conception de la vérité véhiculée par la photographie.

L'association inédite que le photographe fait des conventions du portrait d'enfant et de la représentation d'une réalité sociale très dure - ce que Jacob Riis avait amorcé - lui permet de brouiller les frontières sociales en mêlant deux régimes de visibilité. Ce faisant, il touche un large public. En termes d'efficacité démonstrative, c'est bien parce qu'il cumule la valeur de preuve et une forte charge émotionnelle que ses photos ont un tel pouvoir de mobilisation. Quant aux procédés rhétoriques utilisés, ils atténuent de fait la neutralité supposée du document photographique. Le texte y joue une part prépondérante et ce dernier accompagne des images de plus en plus découpées, tronquées et recontextualisées, qui lui apparaissent soumises mais participent néanmoins toujours du processus démonstratif. Si le texte relève parfois de la simple narration, il est souvent incisif: l'auteur a recours à l'ironie pour interpeller son lecteur, faire appel à sa compassion, ou même parfois le culpabiliser. Lewis Hine 
emprunte aussi à la poésie, dans une démarche créative originale. Mais la méthode la plus surprenante et efficace réside dans l'alliance des images du photographe et de pratiques publicitaires employées par les tenants du camp adverse. L'arsenal utilisé traditionnellement à des fins commerciales sert ici plus que jamais la volonté des Progressistes d'intervenir sur le réel grâce à l'image. La vérité du document, devenue contestable à force de manipulations, cède alors la place à celle du photographe et de ses idéaux politiques et sociaux.

\section{BIBLIOGRAPHIE}

AUBERT, Didier, Photographie et progressisme : The Pittsburgh Survey, 1907-1914, Université Lumière Lyon 2, 2000.

---, « Lewis Hine et les images anonymes du Pittsburgh Survey », Études photographiques, 17, novembre 2005, mis en ligne le 05 décembre 2005, http://etudesphotographiques.revues.org/ index758.html.

---, « Lewis W. Hine : Panneau d'exposition pour le Comité national contre le travail des enfants », in Brunet, François, dir., L'Amérique des images, Histoire et culture visuelles des États-Unis, Paris, Éditions Hazan/Paris Diderot, 2013.

BECK, Tom, « Duality in Lewis Hine's Child Labor Photographs ", Priceless Children: American photographs 1890-1925, Lewis Hine, F. Holland Day: Child Labour and the Pictorialist Ideal, Washington, University of Washington Press, 2001.

CHINN, Sarah E, Inventing Modern Adolescence, The Children of Immigrants in Turn-of-the-Century America, Rutgers University Press, 2008.

DIMOCK, George, « Children of the Mills: Re-Reading Lewis Hine's Child-Labour Photographs, » Oxford Art Journal, 16.2, 1993, 37-54

FINNEGAN, Cara, A., « Studyng Visual Modes of Public Adress: Lewis Hine's Progressive-Era Child Labor Rhetoric ", The Handbook of Rhetoric and Public Address, Shawn J. Parry-Gilles and J. Michael Hogan, dirs., Blackwell Publishing, 2010, 250-70.

GOLDBERG, Vicki, Photography in print: writings from 1816 to the present, Albuquerque, University of New Mexico Press, 1981.

---, Lewis Hine: Children at Work, Munich, London, New York, Prestel Verlag, 1999.

HALES, Peter Bacon, Silver Cities: Photographing American Urbanization, 1839-1939, Albuquerque, University of New Mexico Press, 2005.

HIGONNET, Anne, Pictures of Innocence, the History and Crisis of Ideal Childhood, London Thames \& Hudson, 1998.

HINE, Lewis W, "Social Photography; how the Camera May Help in the Social Uplift ", National Conference on Social Welfare, Official proceedings of the annual meeting, 1909, 355-359, UMDL Texts, University of Michigan Digital Library Text Collections, http://quod.lib.umich.edu/n/ ncosw/ach8650.1909.001/376? page=root;size=100; view=image. 
« High Cost of Child Labor », National Child Labor Committee, June 1914.

ISEMAN, Stephen Dane, Showing as a way of saying: The photograph and word combinations of Lewis Hine in support of child labor reform, Ph.D., The Ohio State University, 1993.

KAPLAN, Daile, « "The Fetish of Having a Unified Thread”: Lewis W. Hine's Reaction to the Use of the Photo Story in Life Magazine ", Exposure, 27:2, 1989, 9-21.

LESME, Anne, « L'enfant pauvre et la naissance de la photographie sociale aux États-Unis au XIXe siècle », E-rea, 7.2 | 2010, 23 novembre 2010, http://erea.revues.org/1237.

---, L'enfant dans la photographie sociale américaine de 1888 à 1941 : enjeux sociaux et esthétiques, Thèse de doctorat, Aix-Marseille Université, 2012.

LESME, Anne, «La photographie sociale : comment l'appareil photo peut contribuer au progrès social» (1909), traduction et commentaire d'un texte de Lewis W. Hine, Art et utopie - pensées anglo-américaines, Mathilde Arrivé, dir., Coll. « Essais sur l'art », Volume 5. Paris, Michel Houdiard, 2012.

« Little Brother », The Survey, vol. 34, May 8, 1915.

LUGON, Olivier, « L'Esthétique du document ", L'Art de la photographie des origines à nos jours, Paris, Citadelles \& Mazenod, 2007, 357-421.

NORDSTROM, Alison, « Lewis Hine », Lewis Hine: de la collection George Eastman House, International Museum of Photography and Film: [exposition], Fondation Henri Cartier-Bresson, 7 septembre - 18 décembre 2011, Alcobendas : TF Editores ; Madrid : Fundacion MAPFRE, 2011, 17-35.

ORVELL, Miles, American Photography,Oxford, Oxford University Press, 2003.

PACE, Patricia, «Staging Childhood: Lewis Hine's Photographs of Child Labor », The Lion and the Unicorn, 26, 2002, The John Hopkins University Press, 324-352.

PERRY, Claire, Young America: Childhood in 19th Century Art and Culture, exhibition, New Haven, Yale University Press, 2006.

«Priceless Children : American photographs 1890-1925, Lewis Hine, F. Holland Day : Child Labour and the Pictorialist Ideal », Washington, University of Washington Press, 2001.

RIIS, Jacob, How the Other Half Lives: Studies Among the Tenements of New York, Charles Scribner \& Sons, 1890 .

SAMPSELL WILLMANN, Kate, " Lewis Hine, Ellis Island, and Pragmatism: Photographs as Lived Experience ", Journal of the Gilded Age and Progressive Era, 7:2, April 2008.

SAMPSELL-WILLMANN, Kate, Lewis Hine as Social Critic, Foreword by Alan Trachtenberg, University Press of Mississipi, 2009.

STANGE, Maren, Symbols of Ideal Life: Social Documentary Photography in America, 1890-1950, New York, Cambridge University Press, 1989.

« Tasks in the Tenements », The Child Labor Bulletin, vol. III, n¹, May 1914.

"The Child's burden in Oyster and Shrimp Canneries », The Child Labor Bulletin, 2, 1913.

The Child Labor Bulletin, 7, February 1915

"Three bits of testimony for the consumers of shrimp and oysters », The Survey, Time Exposures by Hine, Feb. 28, 1914. 
TRACHTENBERG, Alan, « Ever-The Human Document » in America \& Lewis Hine: Photographs, 1904-1940, oreword by Walter Rosenblum; biographical notes by Naomi Rosenblum; essay by Alan Trachtenberg; design by Marvin Israel, Millerton (N.Y.), Aperture Monographs, 1977, 118-42.

---, Reading American Photographs: Images as History, Mathew Brady to Walker Evans, New York, Hill and Wang, 1989.

ZELIZER, Viviana A. Rotman, Pricing the Priceless Child: the Changing Social Value of Children, Princeton (N.J.), Princeton University Press, 1994.

\section{Sources iconographiques:}

Library of Congress, Prints \& Photographs Division, National Child Labor Committee Collection : Fig.1 (LC-DIG-nclc-01607), fig.2 (LC-DIG-nclc-03886), fig.11 (LC-DIG-nclc-04924), fig.12 (LC-DIGnclc-04928), fig.14 (LC-DIG-nclc-04875).

Library of Congress, National Child Labor Committee (U.S.) records, 1904-1953 (bulk 1904-1934) : Fig.3, fig.4, fig.5, fig.6, fig.7, fig.10, fig.15.

Library of Congress, The Survey : Fig.8, fig.9, fig.13.

\section{NOTES}

1. Légende complète : John Huggins. Said he is fourteen years old and has been doffing for eight months in the Guadalupe Valley Cotton Mills. Violation of law. Gets a dollar a day now. Before he came here, he worked in the cotton mill at West, Tex., for five or six years. Said boys work in the Cuero mill under age. « They don't even bother to ask your age. Didn't ask mine. Easy 'nuff to git a job.» The mills were not running on account of floods this week. I found only one other boy under age, «Spider » Estes said he is fourteen years old and been working here, doffing one year. Location: Cuero, Texas. 1913 October.

2. Il convient de noter ici l'existence de voix discordantes, dont celle de George Dimock qui, en s'appuyant sur l'exemple d'une famille « Doffer Family » (GEH $77: 181$ :30) écrit : « However, I find it more plausible to interpret this motif as a sign of the photographer's class dominance vis-à-vis his subjects. Precisely because the mother and children depicted in Doffer Family are lower working-class, Hine is free to pose them as he pleases, positioning them frontally as objectifications of his political project, the fight against child labor. » (Dimock 48)

3. Riis, Jacob, How the Other Half Lives: Studies Among the Tenements of New York, Charles Scribner \& Sons, 1890.

4. Paul Underwood Kellogg (1879-1958), journaliste et réformateur social, a dirigé le Pittsburgh Survey (1907-1908), il fut le rédacteur en chef de la revue progressiste The Survey (1912) et fonda Survey Graphic en 1921. 


\section{RÉSUMÉS}

En photographiant les enfants au travail dans une visée réformatrice de plus en plus affirmée pour le compte du National Child Labour Committee entre 1908 et 1918, Lewis Hine déploie diverses modalités d'interaction du texte et de l'image, convaincu du pouvoir de la photographie de servir la cause d'une information objective. L'intentionnalité du photographe s'appuie sur une conception de la vérité à la fois politique et morale, fruit de son engagement progressiste, et scientifique et technique - il accorde une confiance totale aux qualités intrinsèques du médium. L'objectif de communication qu'il se fixe le pousse à multiplier les méthodes de diffusion de ses photographies, à la recherche d'une efficacité rhétorique toujours plus grande. Cet article s'attache à montrer, à travers l'analyse de différents modes d'organisation des images et du texte, comment le photographe est l'artisan d'une vérité éminemment construite pour guider le lecteur ou le spectateur vers une interprétation toujours plus contrôlée, jusqu'à allier document et pratique publicitaire avec la conception des affiches.

Photographing children at work for the National Child Labor Committee (1908-1918), Lewis Hines uses various methods where text and image interact to communicate his markedly reformist vision. Convinced of the ability of photography to serve the cause of objectivity, the photographer commits himself to a conception of the truth that is at once political and moral, resultant of his engagement with Progressism, as well as his commitment to science and technology-founded on his confidence in the inherent qualities of the medium. Through diversifying and increasing the ways in which he disseminates his photographs, Hines searches for the most effective rhetoric. This article endeavors to show the photographer as the architect of an elaborately constructed truth. It will analyze the different modes he employs in organizing his images in order to guide the reader/viewer towards an interpretation that is increasingly controlled, eventually even linking documentary photography and advertising with poster design.

\section{INDEX}

Keywords : bootblack boy, child labor, icon, National Child Labor Committee (NCLC), newsboy, photography, shoeshine boy, 19th century, 20th century

Mots-clés : enfants au travail, États-Unis, National Child Labor Committee (NCLC), textes et images, photographie, XIXe siècle, XXe siècle 